\title{
IMPROVIng OUTCOMES In STUdent DESIGN COURSES THROUGH QUALITATIVE USER RESEARCH AND CONTEXTUAL IMMERSION
}

\author{
Florin Gheorghe, Antony J. Hodgson, and H.F. Machiel Van der Loos \\ Department of Mechanical Engineering, University of British Columbia \\ florin.gheorghe@alumni.ubc.ca
}

\begin{abstract}
Shifting from the course-based mindset into the real-world context of the user is a challenge that students often face during design courses. This can result in designs and proposed solutions that do not fully meet the technical and business needs of the client.

This paper proposes a greater use of qualitative methods, paired with a deep immersion in the user environment, and highlights the value in design education through a case study example.

A focus on qualitative user-studies in the discovery phase of design helps to give students perspective on the unique characteristics of users and the design context. The Engineers in Scrubs Program, in collaboration with the Uganda Sustainable Trauma Orthopaedic Program (USTOP), at the University of British Columbia is highlighted as one such example.
\end{abstract}

Keywords: Design, Qualitative Methods, Medical Devices, International Surgery, Outcome-Driven Innovation

\section{INTRODUCTION}

Students involved in design courses often find difficulty in switching from the course-based mindset into the real-world context of the user, resulting in designs that do not fully meet the needs of the client. The problem is more prevalent in fields with 'expert-users', such as the work described here in medical device design involving Ugandan and Canadian surgeons. Such perspective shift is increasingly needed as companies and product design are becoming more globalized.

This paper and presentation argues for an increased use of qualitative user research methods, combined with deep contextual immersion of design students in the use environment.

The case study presented here is based on research carried out in collaboration with Canadian and Ugandan surgical teams, a project born out of the Engineers in Scrubs Program at the University of British Columbia (UBC). The aim of the study is to inform how medical device designers may approach innovation differently in low-resource international settings, compared to the familiar Western markets [2]. Understanding design needs for international surgery is particularly difficult, first due to the gap in understanding of the use context between engineers and 'expert-user' surgeons, and second because of the language and cultural barriers to information sharing.

In order to overcome these challenges, a series of qualitative and participatory research methods were introduced early into the design process, and paired with deep contextual immersion of researchers.

\section{METHODOLOGY}

The Engineers in Scrubs Program (EiS) at UBC is a graduate-level course that facilitates design collaborations between engineering students and clinicians. In this program, a series of shadowing exercises and site visits expose students to the challenges faced in medical practice. Clinicians are then invited to propose problems for students to solve during collaborative workshops, and interact closely with students throughout an eight-month design process. By spending time in Canadian hospitals and operating environments throughout the design process, EiS students come to better understand the processes, language, and ways of thinking in surgery, which differ from those in engineering.

The first author in this paper participated in the EiS program and subsequently joined the Uganda Sustainable Trauma Orthopaedic Program (USTOP), a team of orthopaedic surgeons and nurses from UBC and Vancouver General Hospital, on a trip to Mulago National Referral Hospital in Kampala, Uganda. Ethnographies of surgeons and nurses were conducted using observation, interviews, as well as self-guided reflection tools known as cultural probes [1], which included journals and disposable cameras. This data was collected and analyzed using a Grounded Theory approach, a method that uses iterative data collection and sorting, and uncovers emergent patterns in order to build theory around a particular research question or topic [3].

Furthermore, the technique of Outcome-Driven Innovation was used with medical professionals to define the surgical process through the eyes of practitioners, and 
to identify pain points, or difficulties, in the process that warrant innovative solutions [4].

\section{RESULTS AND DISCUSSION}

The Outcome-Driven Innovation (ODI) workshops with users have resulted in 148 unique goals and challenges encountered during a typical surgical procedure. In a second round of data collection, surgeons ranked each one on the level of importance, as well as current satisfaction, narrowing the design space to 10-15 potential opportunities for innovation. These 10-15 highpriority problem areas were then assessed through the cultural and contextual lens that was afforded by the qualitative ethnographic research. Thus, each stand-alone design problem became enveloped in a rich story of contextual, cultural, and user experience on which to base further design decisions.

As an example of these two complementary methods, the ODI results pointed to the problem of fracture reduction, a process of re-aligning the broken bone fragments, as something both demanding of time and especially effort. It was however the qualitative research, shadowing, and interviewing of clinicians that shed a new light on this problem. The reason for the difficulty in reducing fractures in Uganda compared to Canada is that a vast majority of patients present with injury late, and may not be operated on for weeks or months after injury. This opens up the problem space beyond just "how might we decrease time and effort for fracture reduction," but more broadly, "how might we improve reduction of old fractures with significant muscle contracture and atrophy?"

The collaboration between EiS and USTOP has thus far led to student team designs of a low-cost sterilizer device, as well as a unique sterile casing that allows the use of off-the-shelf, low-cost, and non-sterilizable drills to be used in place of highly expensive orthopaedic surgical drills. Further technology design projects are in progress.

\section{Acknowledgements}

The authors would like to thank the Engineers in Scrubs Program, as well as the Branch for International Surgery at the University of British Columbia for their support of this research.

\section{References}

[1] Bill Gaver, Tony Dunne, and Elena Pacenti, "Design: Cultural probes," Interactions, vol. 6, no. 1, pp. 21-29, 1999.

Available as of May 1, 2013 from

http://dl.acm.org/citation.cfm?id=291235

[2] Florin Gheorghe, H.F. Machiel Van der Loos, "Participatory design for surgical innovation in the developing world," in Proc. International Conference on Research into Design, ICoRD'13, Amaresh Chakrabarti, Raghu V. Prakash (ed.) (Chennai, India; 7-9 January 2013), 12 pp., 2013.

Available as of May 1, 2013 from http://link.springer.com/chapter/10.1007/978-81-322-10504_54

[3] Barney G. Glaser, Anselm L. Strauss, The Discovery of Grounded theory: Strategies for Qualitative Research. New Jersey: Transaction Publishers, 1967, 271 pp. \{ISBN: 9780202302607\}

[4] Anthony W. Ulwick, "Turn customer input into innovation," Harvard Business Review, vol. 80, no. 1, pp. 91-97, 2002. 